\title{
ESPECIES DEL COMPLEJO DE JOBOTOS (Phyllophaga spp., Anomala spp. y Cyclocephala spp.) ASOCIADAS A CULTIVOS, EN EL VALLE CENTRAL Y PACÍFICO SECO DE COSTA RICA ${ }^{1}$
}

\author{
Giselle Abarca², Miguel Quesada ${ }^{2}$
}

\begin{abstract}
RESUMEN
Especies del complejo de jobotos (Phyllophaga spp., Anomala spp y Cyclocephala spp.) asociadas a cultivos, en el Valle Central y Pacífico seco de Costa Rica. Se determinaron las especies del complejo de jobotos (Phyllophaga, Anomalay Cyclocephala) asociadas a cultivos y a diferentes grandes grupos de suelos, en algunas zonas agrícolas del Valle Central y Pacífico Seco de Costa Rica, entre 1990 y 1992. El raster y la genitalia masculina fueron micrografiados en un microscopio electrónico de rastreo (MER) y utilizados para la identificación de géneros y especies. Las larvas y adultos identificados fueron $P$. menentriesi, $P$. obsoleta, $P$. zunilensis, $P$. elenans, $P$. vicina y $P$. valeriana. Del género Cyclocephala fueron $C$. lunulata, $C$. castaniella y $C$. sanguinicollis, mientras que en el género Anomala fueron A. cupricollis y A. granulipyga. La distribución por cultivos fue la siguiente: en cafeto (Coffea arabica $\mathrm{L}$.) se encontraron $P$. menentriesi y $P$. vicina; en espárrago (Asparagus officinalis) P. menentriesi; en fresa (Fragaria anannas) P. menentriesi, P. obsoleta, P. zunilensis, Anomala spp., Cyclocephala spp. y $C$. lunulata; en caña de azúcar (Saccharum officinarum) P. zunilensis y P. elenans; en papa (Solanum tuberosum) y en tomate (Lycopersicon esculentum) $P$. valeriana; en pasto kikuyo (Penissetum clandestinum) $P$. obsoleta, C. castaniella, C. sanguinicollis y A. granulipyga. Las larvas de $P$. menetriesi se encontraron siempre en tres grandes grupos de Andisoles: Melanudands, Hapludands y Haplustands. P. obsoleta en el gran grupo Melanudands. $P$. vicina en Hapludands. P. valeriana en Inceptisoles del gran grupo de los Ustropepts. Anomala spp. en Hapludands y Cyclocephala spp.en Haplustands y Melanudands.
\end{abstract}

\begin{abstract}
White grubs complex (Phyllophaga spp., Anomala spp. and Cyclocephala spp.) associated to agricultural crops of the Central Valey and Dry Pacific of Costa Rica. During 1990 and 1992 speciesofthe white grubs complex Phyllophaga, Anomala and Cyclocephala) were found associated to agricultural crops and to different groups of soils, in cultivated arcas of the Central Valley and Dry Pacific of CostaRica. White grubs raster and male genital, observed with the scanning electron microscope (SEM), were used to identify genera and species. Larvae and adults were identified as Phyllophaga menetriesi, P. obsoleta, P. zunilensis, P. elenans, $P$. vicina and $P$. valeriana; Cyclocephala lunulata, $C$. castaniella and C. sanguinicollis; and Anomala cupricollis and A. granulipyga. The distribution in agricultural crops was: $P$. menetriesi and $P$. vicina on coffee (Coffea arabica); $P$. menetriesi on asparagus (Asparagus officinalis); P. menetriesi, P. obsoleta, P. zunilensis, Anomala spp., Cyclocephala spp., Cyclocephala lunulata on strawberry (Fragaria anannas); $P$. zunilensis, $P$. elenans on sugar cane (Saccharum officinarum); $P$. valeriana on potatoe (Solanum tuberosum) and tomatoe (Lycopersicon esculentum); P. obsoleta, C. castaniella, C. sanxuinicollis and A. granulipyga on kikuyu grass (Penissetum clandestinum) P. menetriesi larvae were found in Andisols grand groups: Melanudands, Hapludands and Haplustands. $P$. obsoleta in the Melanudands grand group. $P$. vicina in Hapludands. $P$. valeriana in the Inceptisols grand group of Ustropepts. Anomala spp. in Hapludands and Cyclocephala spp. in Hapludands and Melanudands.
\end{abstract}

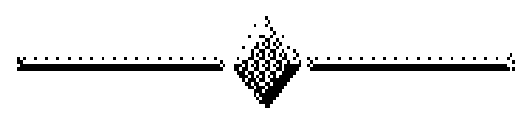

\section{INTRODUCCIÓN}

El complejo de larvas escarábidas, conocido popularmente en Costa Rica con el nombre de jobotos o con los de fogatas y gallina ciega en Centroamérica, está formado principalmente por especies del género Phy- llophaga, mientras que las de los géneros Anomala y Cyclocephala son consideradas de menor relevancia (Abarca, et al., 1992; Marón, 1986).

En Centroamérica se ha informado aisladamente sobre identificación, aspectos de la biología y

\footnotetext{
1 Centro de Investigaciones en Protección de Cultivos (CIPROC), Escuela de Fitotecnia, Facultad de Agronomía, Universidad de Costa Rica. San José, Costa Rica.

2 Laboratorio de Entomología, Escuela de Fitotecnia, Facultad de Agronomía, Universidad de Costa Rica. San José, Costa Rica.
} 
comportamiento de estos escarábidos, que ocasionan daño al sistema radical de una gama amplia de cultivos anuales y perennes. En Costa Rica se ha informado de algunas especies como $P$. menetriesi, $P$. vicina, $P$. obsoleta, $P$. elenans, $P$. hondura, $P$. parvisetis, $P$. orosina, $P$. crinalis, $P$. densata, $P$. gigantea, $P$. pruinosa, $P$. costarricensis y $P$. prolixa (Coto, 1993). No obstante, para encontrar una estrategia de manejo de estos insectos plaga, es necesario estudiar su biología, distribución y huéspedes alternativos; es conveniente mencionar que existen muy pocos registros confiables de observaciones que nos permitan establecer una relación específica entre planta e insecto (Morón, 1986).

Por la poca información local sobre este insectoplaga, se realizó un estudio cuyo objetivo fue identificar las especies de jobotos presentes en algunas zonas agrícolas de Costa Rica, con el fin de obtener datos más precisos sobre las especies y su asociación a cultivos, ya que no existe base para pensar que todas las especies de jobotos respondan igual ante los métodos de control utilizados contra ellos. Por el contrario, es posible que la dificultad para su combate deriva de su adaptación específica a las modificaciones del microhabitat, integrado por las características genéticas de la planta huésped, la humedad, la composición del suelo, los compuestos tóxicos incorporados y la presencia de otras especies de insectos rizófagas (Morón, 1986).

\section{MA TERIALES Y MÉTODOS}

El presente estudio fue llevado a cabo en las siguientes localidades de Costa Rica: Fraijanes, Provincia de Alajuela, a una altitud de 1800 msnm; entre 1990 y 1992 Y en otras zonas como: San José, Provincia de Alajuela a una altitud de 840 msnm; en San Pablo, Santo Domingo, Vara Blanca, San José de la Montaña, Provincia de Heredia, a altitudes entre 1804 y 1350 msnm; en Juan V iñas, El Tejar, Provincia de Cartago, a altitudes de 1165 y 1400 msnm, respectivamente y en Carrillo, Provincia de Guanacaste, a una altitud de $40 \mathrm{msnm}$. Fraijanes es una región agrícola, donde los cultivos más comunes son: pasto kikuyo (Pennisetum clandestinum), fresa (Fragaria anannas) y plantas ornamentales; mientras que en los otros sitios incluidos en este estudio las tierras se encontraban sembradas principalmente por papa (Solanum tuberosum), tomate (Lycopersicum esculentum), cafeto (Coffea arabica), espárrago (Asparagus officinalis) y caña de azúcar (Saccharum officinarum).

\section{Recolección e identificación de larvas}

En Fraijanes se hizo un muestreo detallado de larvas de jobotos cada ocho días, entre marzo y noviembre y cada 15 días entre diciembre y febrero de cada año, en este último los muestreos fueron hechos a intervalos más espaciados debido a que en este periodo las poblaciones de jobotos disminuyen. En otras zonas del Valle Central y en el Pacífico Seco estos muestreos fueron hechos a intervalos de 30 días, entre mayo y setiembre.

Para la recolección de los jobotos fueron tomadas 10 muestras de suelo distribuídas aleatoriamente por cada $3.500 \mathrm{~m}^{2}$, excavando fosas de $1 \mathrm{~m}$ de largo, 0,30 $\mathrm{m}$ de ancho y $0,70 \mathrm{~m}$ de profundidad; el volumen de suelo obtenido fue tamizado a través de una malla cuadriculada de $1 \mathrm{~cm}$ por $1,5 \mathrm{~cm}$. Las larvas fueron separadas en los estadios L1, L2, L3, y trasladadas al laboratorio. Luego, las poblaciones de los estados inmaduro $\mathrm{s}$ L1 y L2 y una parte de la población L3, fueron colocadas individualmente en envases plásticos rellenos con suelo rico en materia orgánica y sembrados con maíz germinado, con la finalidad de criarlas hasta obtener adultos para confirmar la identificación de las larvas. La otra parte de la población L3 fue fijada en KAAD (etanol, ácido acético glacial, dioxano y kerosene en una proporción de 7:2:2:1, respectivamente) durante ocho horas. Seguidamente, fueron colocados en etanol al $70 \% \mathrm{v} / \mathrm{v}$, para su posterior identificación. El raster y las estructuras taxonómicas observadas en el estadio L3 de las larvas y propuestas por King (1985) y Morón $(1986,1988)$ fueron la base para la identificación a nivel de género y especie.

Una vez identificadas las larvas, se procedió a cortar los últimos segmentos abdominales para realizar un estudio más detallado del raster. Parala limpieza de esta estructura estos segmentos abdominales fueron sumergidos en agua destilada y limpiados de los restos de tejidos del intestino conla ayuda de pinzas cntomológicas, agujas de disección y pinceles; luego fueron colocados en etanol70\% v/v durante 2 horas, posteriormente en una solución de $\mathrm{KOH}$ al $10 \%$ por 30 seg. y finalmente en agua destilada para finiquitar la limpieza. Después fueron lavados dos veces en solución Carl, mantenidos en esta misma solución durante 24 horas y luego deshidratados en una serie graduada de etanol (70-80-85-90-95-100-100-100\% de etanol v/v), a 21 $23^{\circ} \mathrm{C}$, con intervalos de $15 \mathrm{~min}$. entre cada paso. Una vez que los segmentos abdominales estaban en la última etapa con alcohol absoluto, fueron colocados en una pequeña cámara plástica con tapas de malla fina e inmediatamente fueron secados en un secador de punto crítico con siete cambios de $\mathrm{CO}_{2}$ de 5 min. de duración cada uno. Los segmentos abdominales fueron montados en posición dorsal sobre la superficie engomada de una platina de aluminio. Todas las estructuras taxonómicas de estos segmentos fueron recubiertas con platino en un cobertor iónico GIKO IB-5 y observadas en un microscopio electrónico de rastreo (MER) a $15 \mathrm{Kv}$ de voltaje 
acelerador (Grodowitz, et al., 1982; López y Salazar., 1987).

\section{Captura e identificación de adultos}

En la Estación Experimental Fabio Baudrit Moreno, subestación Fraijanes, de la Universidad de Costa Rica y en la finca de un agricultor ubicadas en la zona de estudio, fueron hechas capturas de adultos con trampas de luz negra de $40 \mathrm{~W}$, suspendidas en postes fijos a una altura de $2 \mathrm{~m}$ y encendidas durante toda la noche. Estas capturas fueron hechas entre el15 de enero de 1990 y el 31 de julio de 1992. Los recuentos de los adultos capturados fueron realizados por las mañanas, a intervalos de ocho días entre abril y agosto y cada 15 días de setiembre a marzo, en éste último los recuentos se distanciaron más en el tiempo debido a que la población de adultos disminuyó.

Los adultos colectados fueron trasladados al laboratorio, identificados con un número, preservados y montados en alfi leres entomológicos de acuerdo a las normas de preservación de insectos (Morón y Terrón, 1988). Posteriormente fueron clasificados a nivel de género con las claves elaboradas por Morón (1988); y la identificación de especies fue basada en la genitalia masculina y las estructuras taxonómicas observadas en los adultos, según lo propuesto por Morón (1986). Una vez identificados los adultos hasta especie, se procedió a preparar las genitalias masculinas para tomar las micrografías de los detalles en el MER. Los adultos ma- chos fueron colocados en agua a punto de ebullición durante 3 minutos, con el fin de suavizar los tejidos; luego se procedió a extraer la genitalia y colocada en un plato petri con agua destilada para realizar la limpieza preliminar de la misma. En seguida, fueron sumergidas en una solución de KCL al $10 \%$ por 15 seg. y finalmente se les lavó con agua destilada para terminar la limpieza. Posteriormente, se siguieron todos los pasos anteriormente descritos en la preparación de la muestra para ser observadas con el MER.

\section{RESULTADOS Y DISCUSIÓN}

\section{Especies de jobotos predominantes}

Las larvas y adultos colectados en las zonas de estudio fueron identificados como pertenecientes a tres géneros a: Phyllophaga, Anomala y Cyclocephala. Las seis especies de Phyllophaga fueron: P. menetriesi, $P$. obsoleta, P. zunilensis, $P$. elenans, $P$. vicina y $P$. valeria$n a$. Las especies de Cyclocephala fueron $C$. lunulata, C. castaniella y C. sanguinicollis; mientras que en Anomala fueron A. cupricollis y A. granulipyga.

Los estados inmaduros de Phyllophaga fueron reconocidos principalmente por la forma de "V" o de "Y" en la abertura anal, así como por el raster con su palidia bien clesarrollada (Figura 1); en Anomala y Cyclocephala la abertura anal presentó forma semi circular o transversal, además de que Cyclocephala tiene un

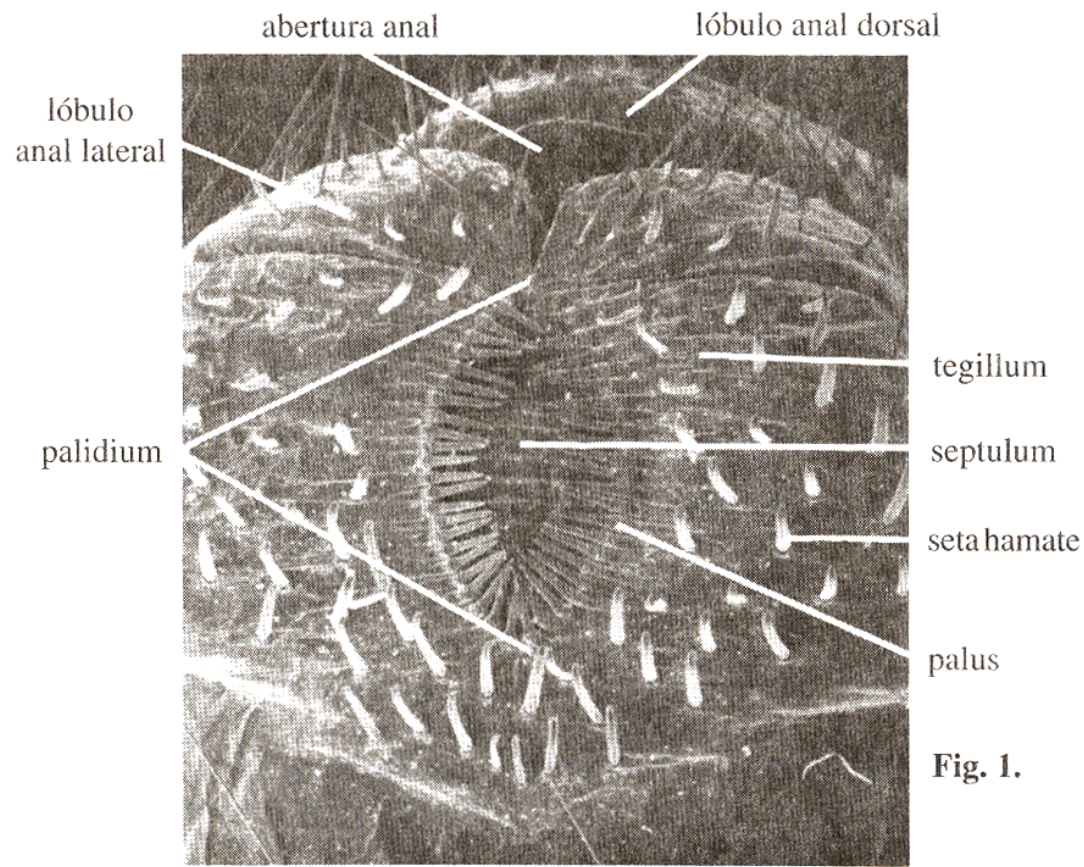

Fotomicrografía del raster mostrando detalles de las microestructuras, en larvas $\mathrm{L}_{3}$ de Phyllophaga spp., a 35X. 
raster sin palidia (Figuras 2a,b,c,d); lo,anterior concuerda con lo anotado por King (1984).

Las especies de Phyllophaga fueron reconocidas principalmente por la forma de la palidia, por ejemplo, en $P$. menetriesi fueron observados palis de $22 \pm 5$ por palidia, colocados muy cerca uno del otro, los que en su mayoría son aplanados dorso ventralmente; con más de 40 setas hamate por tegila (Figuras 3b,d). En P. vicina el raster presentó la palidia larga e irregular, con palis entre 22 y $25 \pm 5$ de tamaño corto, con las puntas encorvadas y separadas por una distancia igual o más grande que la base del pali, los que se traslapan en los extremos de las palidias, la septula es más ancha cerca de la mitad de la palidia (Figuras. 3a,c); en P. elenans se observó un raster con palidias bien desarrolladas, cuyos palis de diferentes tamaños fueron encontrados espaciados irregularmente, con la septula más ensanchada cerca de la parte anterior final de la palidia (Figuras. 4 a,b); en $P$. ohsoleta el raster presentó la palidia con 20 o menos palis, no encorvados en las puntas, separados entre sí por una distancia igualo más corta que la base del pali, y 30 o menos setas hamate por tegila
(Figuras. 4 c,d). En $P$. valeriana se observó un raster con palidias bien desarrolladas y curvadas, con palis de tamaño corto, 16 a 20 por palidia, no encorvados en las puntas y muy cerca uno del otro, la septula es de forma ovalada (Figuras. 4 e,f). Todas estas características concuerdan con lo informado por King (1984).

Los adultos de estos escarábidos fueron separados en géneros y especies con base en las características de la genitalia del macho, de modo que en las Figuras 5, 6 y 7 son ilustradas las diferentes formas del edeago, ornamentos del edeago, falobase, parámetro, pie basal y tecto de cada una.

\section{Complejo de jobotos y las zonas de vida}

Las larvas del complejo de jobotos colectadas en la Provincia de Alajuela, cantón Alajuela, distrito de Fraijanes, cuya zona de vida es Bosque Muy Húmedo Montano Bajo (Tosi, 1969), fueron identificadas como P. menetriesi, P. obsoleta y P. zunilensis (Cuadro 1). En esta misma provincia, en el cantón Alajuela, distrito de San
Fig. 2. Fotomicrografías del raster de larvas $\mathrm{L}_{3}$ de Anomala spp., abertura anal a $30 \mathrm{X}$ y palidia 60C. C-D) Cyclocephala spp., raster sin palidia $30 \mathrm{X}$ y abertura anal $35 \mathrm{X}$.
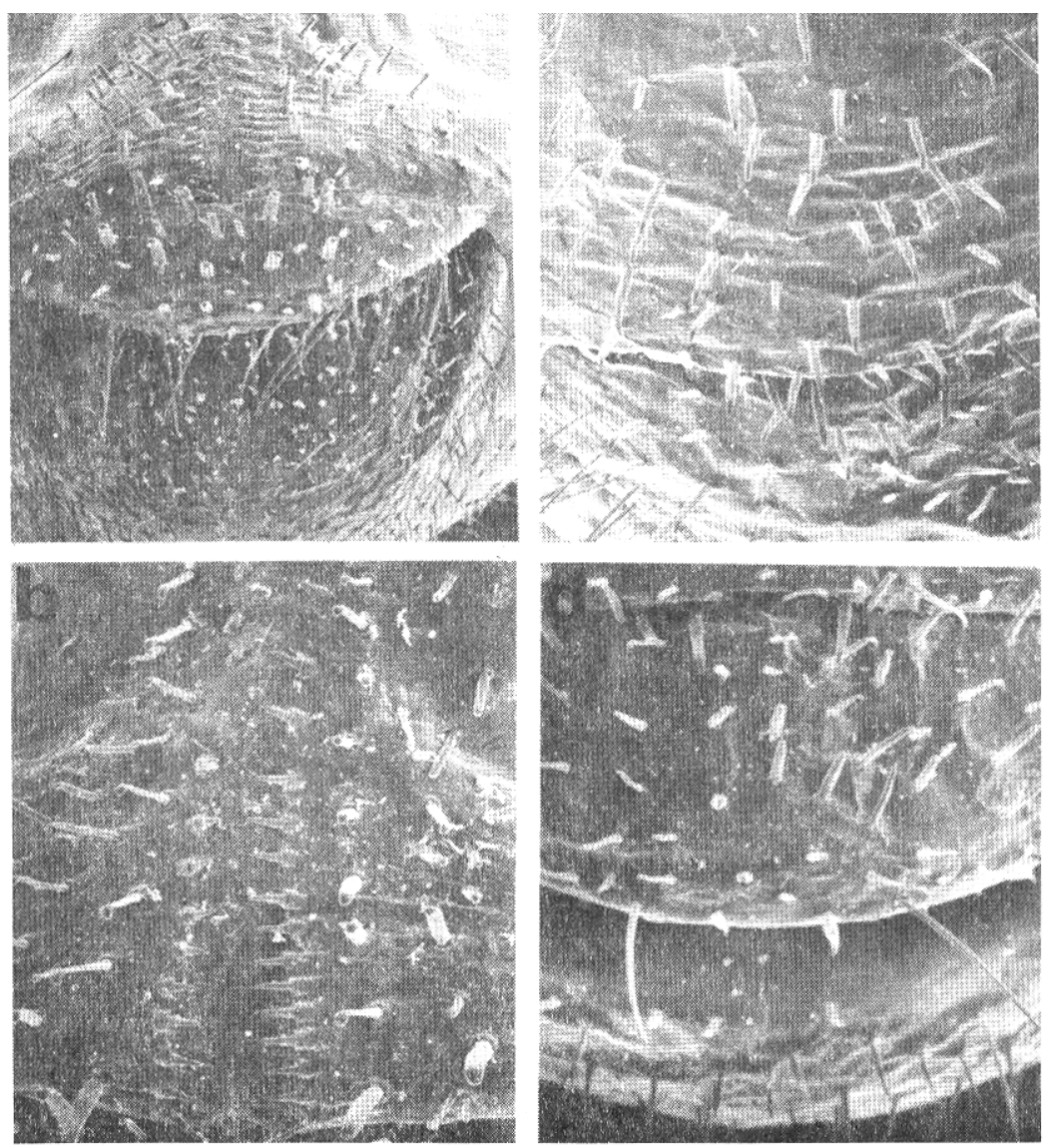
Fig. 3. Micrografías del raster de larvas $\mathrm{L}_{3}$ (Phyllophagaspp., A,C) (P. vicina, palidia a 30X y $40 \mathrm{X}$. B, D) P. menetriesi, palidia a $35 \mathrm{X}$ y $60 \mathrm{X}$.
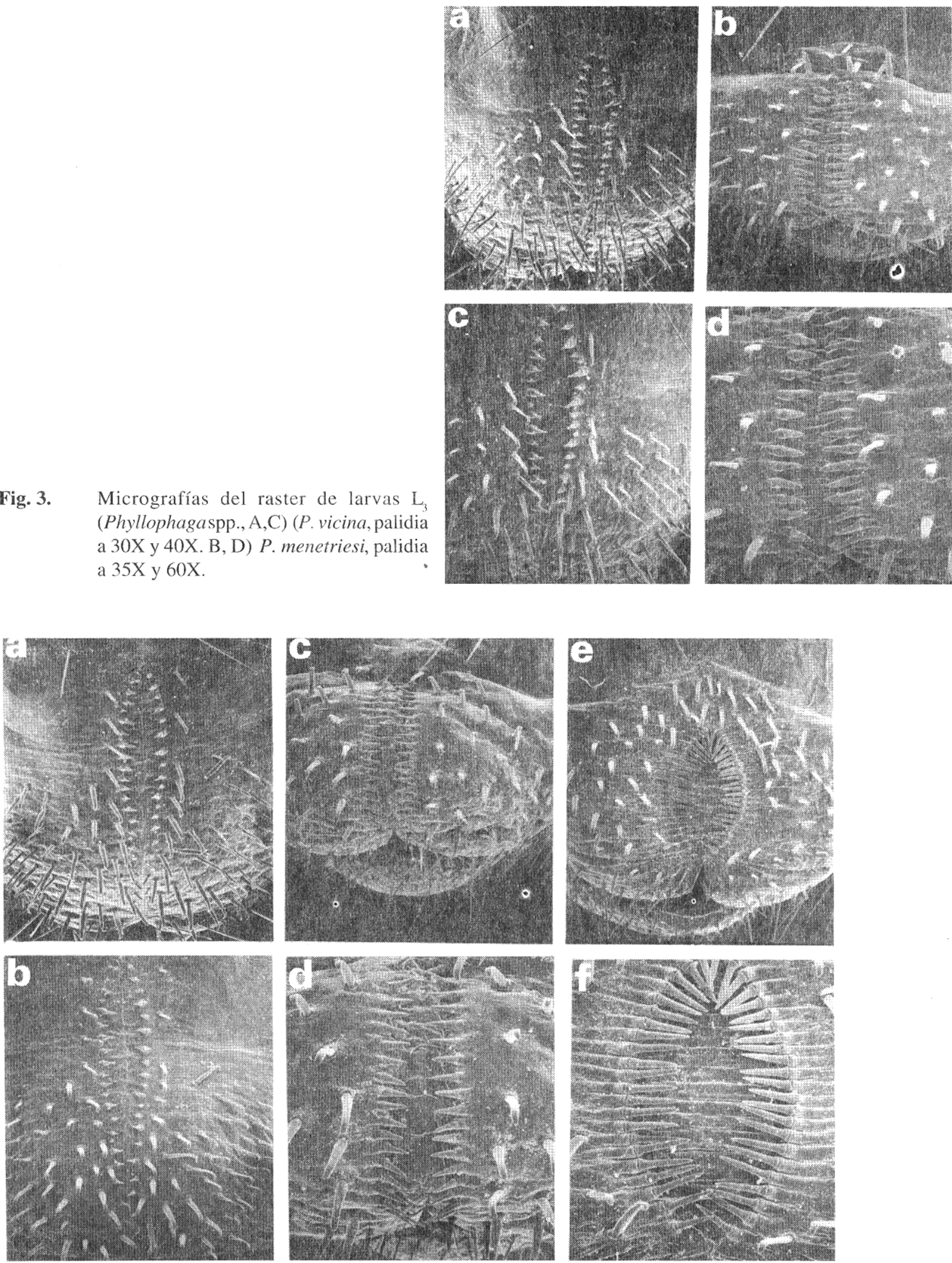

Fig. 4. Fotomicrografías detallando rasters y palidia (Phyllophaga spp., A,C) (P. elenans 30X y 35X. C, D) ( P. obsoleta, 35X y 60X, E,F). P. valeriana 35X y 80X. 
José, con una zona de vida Bosque Pluvial Montano Bajo (Tosi, 1969), fueron identificados como P. menetriesi y Cyclocephala spp. en este caso no fue posible identificar a nivel de especie debido a la falta de claves. En la Provincia de Heredia, cantón de Barba, en los distritos de San Pablo (Buena Vista) y San José de la Montaña con zonas de vida, Bosque Premontano Muy Húmedo y Bosque Muy Húmedo Montano Bajo, respectivamente (Tosi, 1969), los especímenes fueron clasi ficados como Anomala. La identificación de especies no fue posible por carencia de claves; en esta misma provincia, cantón de Heredia, distrito de Vara Blanca con una zona de vida de Bosque Pluvial Montano Bajo (Tosi, 1969) los escarábidos fueron identificados como C. lunulata y en la misma provincia, cantón de Santo Domingo con una zona de vida de Bosque Húmedo Premontano (Tosi, 1969) y en el distrito de San Pablo con una zona de vida Bosque Premontano Muy Húmedo (Tosi, 1969), los coleópteros se clasificaron como P. menetriesi. En la Provincia de Guanacaste, cantón de Carrillo con la zona de vida Bosque Seco Tropical - Transición a Húmedo (Tosi, 1969), los jobotos encontrados fueron identificados

Cuadro 1. Relación entre algunas localidades agrícolas del Valle Central y Pacífico Seco, altitudes y zonas de vida cor las especies del complejo de larvas de jobotos, Costa Rica. 1990-1992.

\begin{tabular}{|c|c|c|c|}
\hline Género y especie & Localidad agrícola & $\begin{array}{l}\text { Altitud } \\
\text { msnm }\end{array}$ & Zonas de vida \\
\hline Phyllophaga menetriesi & $\begin{array}{l}\text { Fraijanes } \\
\text { Prov. de Alajuela } \\
\text { San José } \\
\text { Prov. de Alajuela. }\end{array}$ & 1800 & $\begin{array}{l}\text { Bosque muy Húmedo Montano Bajo } \\
\text { Bosque Pluvial Montano Bajo }\end{array}$ \\
\hline San Pablo & $\begin{array}{l}1361 \\
\text { Prov. de Heredia. }\end{array}$ & & Bosque Premontano Muy Húmedo \\
\hline Santo Domingo & $\begin{array}{l}1350 \\
\text { Prov. de Heredia. }\end{array}$ & & Bosque Húmedo Premontano \\
\hline Phyllophaga obsoleta & $\begin{array}{l}\text { Fraijanes } \\
\text { Prov. de Alajuela. }\end{array}$ & 1800 & Bosque muy Húmedo Montano Bajo \\
\hline Phyllophaga zunilensis & $\begin{array}{l}\text { Fraijanes } \\
\text { Prov. de Alajuela. } \\
\text { Carrillo } \\
\text { Prov. de Guanacaste }\end{array}$ & $\begin{array}{l}1800 \\
40\end{array}$ & $\begin{array}{l}\text { Bosque muy Húmedo Montano Bajo } \\
\text { Bosque Seco Tropical-Transición a } \\
\text { Húmedo }\end{array}$ \\
\hline Phyllophaga elenans & $\begin{array}{l}\text { Carrillo } \\
\text { Prov. de Guanacaste }\end{array}$ & 40 & $\begin{array}{l}\text { Bosque Seco Tropical-Transición a } \\
\text { Húmedo }\end{array}$ \\
\hline Phyllophaga vicina & $\begin{array}{l}\text { Juan Viñas } \\
\text { Prov. de Cartago }\end{array}$ & 1165 & Bosque Húmedo Premontano \\
\hline Phyllophaga valeriana & $\begin{array}{l}\text { El Tejar } \\
\text { Prov. de Cartago }\end{array}$ & 1400 & Bosque Húmedo Premontano \\
\hline Cyclocephala spp. & $\begin{array}{l}\text { San José } \\
\text { Prov. de Alajuela. }\end{array}$ & 840 & Bosque Pluvial Montano Bajo \\
\hline Cyclocephala lunulata & $\begin{array}{l}\text { Vara Blanca } \\
\text { Prov. de Heredia. }\end{array}$ & 1804 & Bosque Pluvial Montano Bajo \\
\hline Anomala spp. & $\begin{array}{l}\text { San Pablo } \\
\text { Prov. de Heredia. } \\
\text { San José de la } \\
\text { Montaña. } \\
\text { Prov. de Heredia. }\end{array}$ & $\begin{array}{l}1361 \\
1520\end{array}$ & $\begin{array}{l}\text { Bosque Premontano Muy Húmedo } \\
\text { Bosque muy Húmedo Montano Bajo }\end{array}$ \\
\hline
\end{tabular}


Cuadro 2. Especies del complejo de adultos de jobotos (Col: Scarabaeidae), (Phyllophaga spp., Anomala spp. y Cyclocephala spp.) relacionadas con algunos cultivos agrícolas, en el Valle Central y Pacífico Seco de Costa Rica. 1990-1992.

\begin{tabular}{|c|c|c|}
\hline Género y especie & Localidades & Cultivos \\
\hline Phyllophaga menetriesi & $\begin{array}{l}\text { Fraijanes, Santo Domingo, } \\
\text { San Pablo, San José }\end{array}$ & $\begin{array}{l}\text { Fragaria anannas } \\
\text { Coffea arabica, Asparagusofficinalis }\end{array}$ \\
\hline Phyllophaga obsoleta & $\begin{array}{l}\text { Fraijanes, San Rafael, } \\
\text { Platanillo, San Antonio }\end{array}$ & $\begin{array}{l}\text { Fragaria anannas, Penissetumm } \\
\text { clandestinum }\end{array}$ \\
\hline Phyllophaga zunilensis & Fraijanes, Carrillo & $\begin{array}{l}\text { Fragaria anannas, } \\
\text { Saccharumofficinarum }\end{array}$ \\
\hline Phyllophaga elenans & Carrillo & Saccharum officinarum \\
\hline Phyllophaga vicina & Juan Viñas & Coffea arabica \\
\hline Phyllophaga valeriana & El Tejar & Solanum tuberosum \\
\hline Cyclocephala spp & San José & Asparagus officinalis \\
\hline Cyclocephala lunulata & Vara Blanca & Fragaria anannas \\
\hline Cyclocephala castaniella & Fraijanes & $\begin{array}{l}\text { Fragaria anannas, Penissetum } \\
\text { clandestinum }\end{array}$ \\
\hline Cyclocephala sanguinicollis & Fraijanes & $\begin{array}{l}\text { Penissetum clandestinum, } \\
\text { Fragaria anannas }\end{array}$ \\
\hline Anomala spp & $\begin{array}{l}\text { San Pablo, } \\
\text { San José de la Montaña }\end{array}$ & Fragaria anannas \\
\hline Anomala cupricollis & Fraijanes & Fragaria anannas \\
\hline Anomala granulipyga & Fraijanes & $\begin{array}{l}\text { Fragaria anannas, Penissetum } \\
\text { clandestinum }\end{array}$ \\
\hline
\end{tabular}
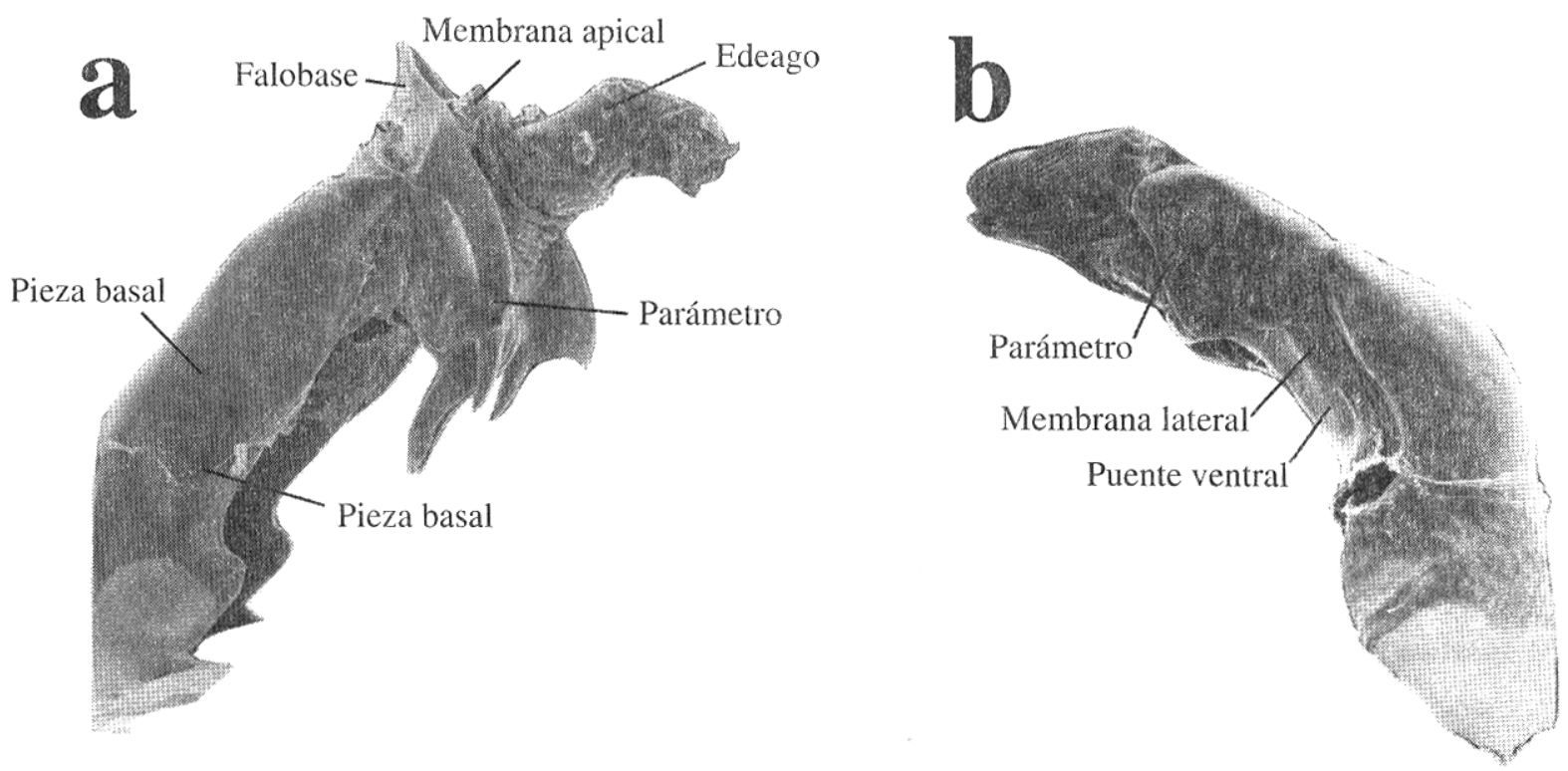

Fig. 5. Micrografías de genitalias masculinas, vistas latero-ventrales. A)Anomala cupricollis 17X. B) Phyllophaga spp. a 17X. 

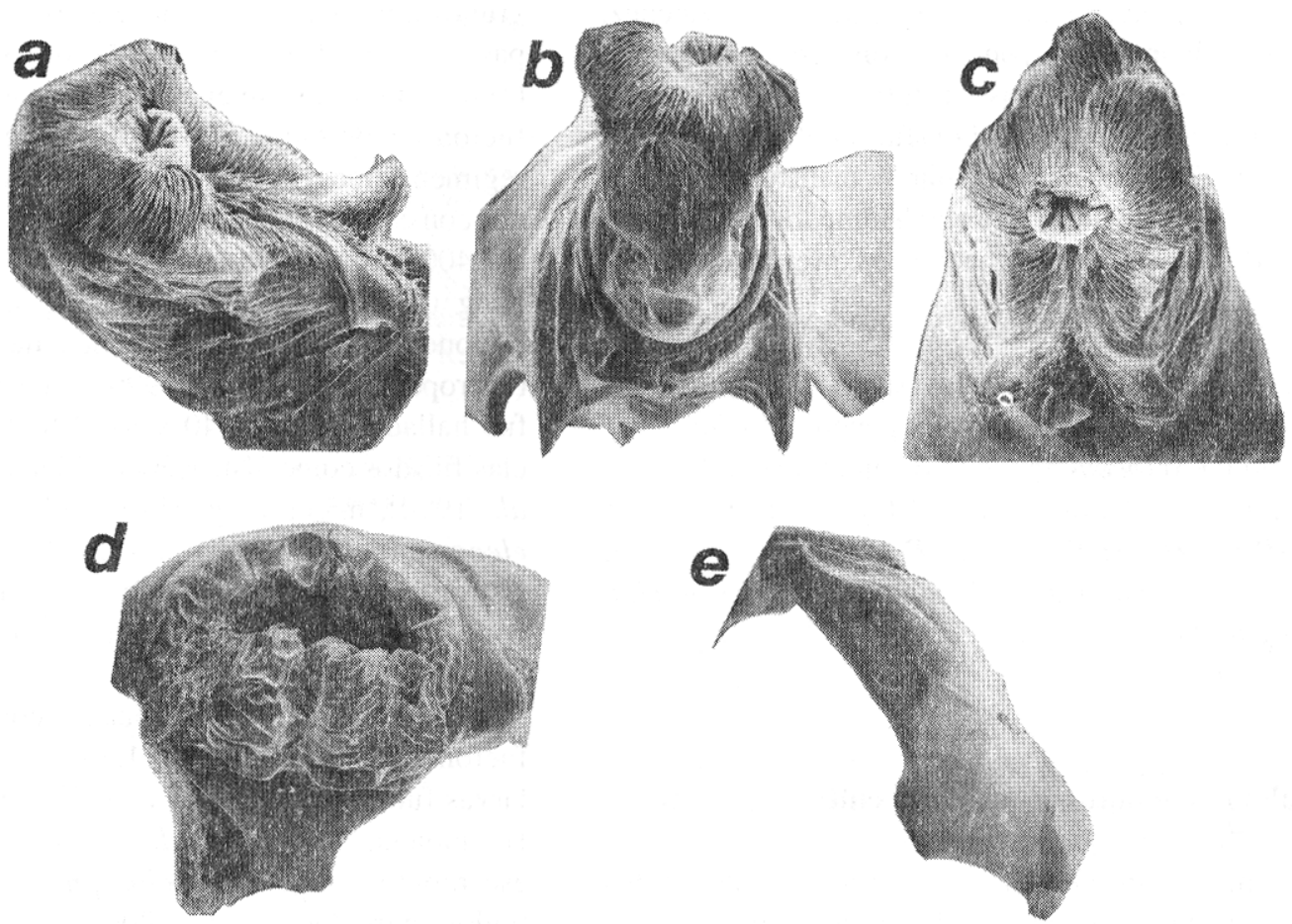

Fig. 6. Fotomicrografías de genitalias masculinas de Phyllophaga spp. P. menetriesi; A) Vista dorso-lateral de los ornamentos del edeago a 40X; B) Vista frontal de la genitalia a 40X, C) Vista frontal de los ornamentos del edeago a 40X. P. obsoleta; D) Vista frontal mostrando ornamentos del edeago a 50X; E) Vista latero-dorsal de la genitalia a $25 \mathrm{X}$.

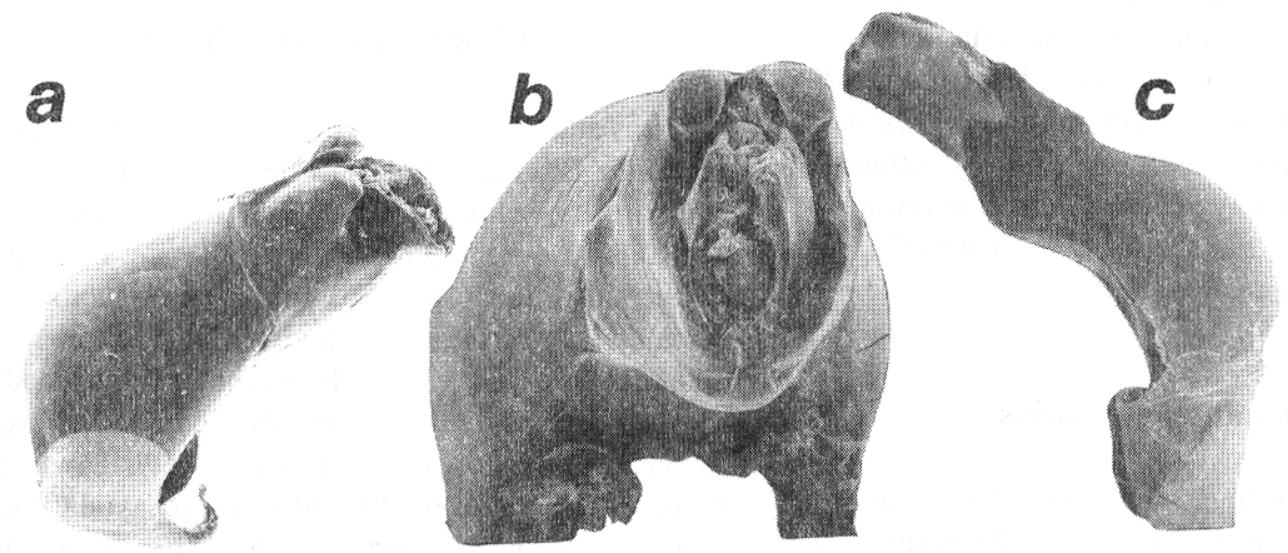

Fig. 7. Micrografías de genitalias masculinas, de Anomala cupricollis A) Vista latero-dorsal a 25X. B) vista frontal a 50X. C) Vista lateral a 22X. D) Vista dorso-frontal del edeago con sus ornamentos a $60 \mathrm{X}$. D) Vista latero-ventral a $17 \mathrm{X}$.

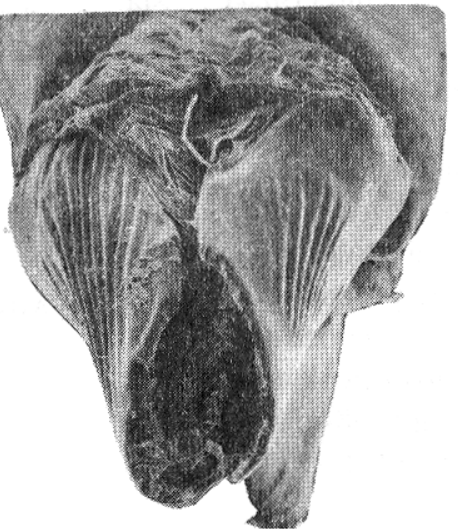

e

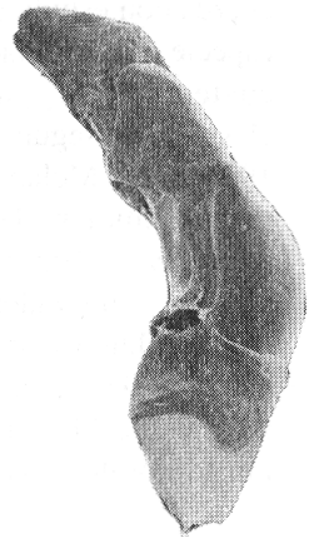


como P. zunilensis y P. elenans. En la Prov. de Cartago, cantón de Jiménez, distrito de Juan Viñas con una zona de vida Bosque Húmedo Premontano (Tosi, 1969), fue encontrada $P$. vicina, mientras que $P$. valeriana fue identificada en esta misma provincia, cantón El Guarco, distrito de El Tejar con una zona de vida Bosque Húmedo Premontano (Tosi, 1969); esta información es presentada en el Cuadro 1.

Los adultos obtenidos de las crías de laboratorio y de las trampas de luz pertenecían a los géneros Phyllophaga, Anomala y Cyclocephala, lo que coincidió con la identificación de las larvas (Cuadros 1 y 2). Las especies fueron $P$. menetriesi, $P$. obsoleta, $P$. zunilensis, $P$. elenans, $P$. vicina, $P$. valeriana, $C$. lunulata, $C$. castaniella, C. sanguinicollis; A. cupricollis y A. granulipyga (Cuadro 2).

\section{Complejo de jobotos asociados a cultivos}

Las nueve especies del complejo de larvas de jobotos fueron colectadas en varios cultivos cuyas poblaciones ocasionaron disminuciones en los rendimientos entre el $20 \%$ y $80 \%$. Por ejemplo en cafeto (Coffea arabica L.) fueron identificadas $P$. menetriesi y $P$. vici$n a$; en espárrago (Asparagus officinalis) fue encontrada P. menetriesi; en fresa (Fragaria anannas) fueron halladas P. menetriesi, P. obsoleta, P. zunilensis, Anomala spp., Cyclocephala spp. y $C$. lunulata; en caña de azúcar (Saccharum officinarum) fueron identificadas $P . z u$ nilensis y $P$. elenans; en papa (Solanum tuberosum) y en tomate (Lycopersicon esculentum) fue encontrada $P$. valeriana; en pasto kikuyo (Penissetum clandestinum) fueron halladas $P$. obsoleta, $C$. castaniella, $C$. sanguinicollis y A. granulipyga.

\section{Complejo de jobotos, altitudes y suelos}

Los especímenes de $P$. menetriesi fueron hallados entre los 840 y 1.800 m.s.n.m. (Cuadro 1), lo que concuerda con estudios realizados por King (1984). Esta especie fue asociada a suelos volcánicos, con altos contenidos de materia orgánica y bien drenados, clasificados según la Taxonomía de Suelos (S.S. Staff, 1994) como Melanudands y Hapludands en los sitios de mayor altura y Haplustands en los de menor altitud (Acon, et al., 1990). También se encontró relacionada a terrenos cultivados con fresa, café y espárrago (Cuadro 2). Los adultos y larvas de $P$. obsoleta fueron encontrados a los 1.800 m.s.n.m. (Cuadro 1), lo que coincide con los trabajos de Marón (1988) y King (1984); los estados inmaduros de esta especie fueron hallados en Andisoles del gran grupo Melanudands (Acon, et al.,
1990). Este grupo de escarábidos estaba relacionado a cultivos como pastos y fresa, lo que concuerda con lo citado por Marón (1988). Los especímenes identificados como $P$. vicina fueron encontrados a $1.165 \mathrm{msnm}$ en Andisoles del régimen údico (Hapludands) (Acon,et al., 1990) cultivados con cafetos. Las larvas P. valeria$n a$ fueron halladas a 1.400 m.s.n.m., lo que concuerda con el estudio de King (1984) Y estaban asociadas a Inceptisoles, de regiones cálidas y con régimen de humedad ústico (Ustropepts) (Acon et al., 1990). Laespecie P. zunilensis fue hallada entre los 40 y los $1.800 \mathrm{msnm}$ en suelos clasificados corno Ustropepts y Melanudands (Acon et al., 1990), mientras que los estados inmaduros de $P$. elenans fueron localizados a $40 \mathrm{msnm}$, en suelos $\mathrm{U}$ stropepts, correspondiendo los Inceptisoles con las zonas bajas y los Andisoles con las zonas altas (Acon,et al., 1990).

Los coleópteros identificados como Anomala spp. fueron localizados entre los 1.361 y 1.520 m.s.n.m. Las larvas fueron encontradas en suelos clasificados como Hapludands (Acon, et al., 1990); mientras que los escarábidos del género Cyclocephala spp. fueron encontrados entre los 840 y 1.804 m.s.n.m. Los estados inmaduros de este género fueron hallados en suelos volcánicos clasificados como Haplustands y Melanudands (Acon, et al., 1990).

\section{AGRADECIMIENTO}

Los autores agradecen a Angel Salís (Instituto Nacional de Biodiversidad, San José, Costa Rica) por su colaboración en la identificación de los adultos de jobotos. A Róger López (Q.D.G.) (Escuela de Fitotecnia, Universidad de Costa Rica) por su contribución en la toma de las micrografías y revisión de este trabajo. A Rafael Mata (Escuela de Fitotecnia, Universidad de Costa Rica) por su colaboración en la clasificación taxonómica de los suelos y por la revisión de este manuscrito. A Justo Azofeifa por su ayuda con el revelado de las micrografías. A la Vicerrectoría de Investigación, de la Universidad de Costa Rica, por la cooperación en el desarrollo de este estudio.

\section{LITERATURA CITADA}

ABARCA,G.;VARGAS,E.;MATA,R. 1992. Alternativas de combate del complejo de larvas de jobotos Phyllophaga spp., Anomala spp. y Cyclocephala spp.) (Col: Scarabaeidae) en fresa (Fragaria ananassa). Agronomía Costarricense 16(1):45-54. 
ACON, J.; MATA, R.; UGALDE, M.; BRICEÑO, L. 1990 Mapa de Suelos de Costa Rica, Esc. 1:200.000. Convenio MAG - SEPSA - MIDEPLAN.

COTO, D. 1993. Morfología de la cápsula genital masculina de especies del género Phyllophaga (Col: Sacarabaeidae). Manejo Integrado de Plagas. Costa Rica. 16: 2830

GRODOWITZ,M.J.; KRCHMA,J.; BROCE,AB. 1982. A method for preparings of bodied larval Diptera for Scanning Electron Microscopy. Journal of the Kansas Entomological Society 55(4):751-753.

HERRERA, W. 1986. Clima de Costa Rica. EUNED. San José, Costa Rica. 118 p. Mapas esc. $1: 250.000$.

KING, AB.S. 1984. Biology and identification of white grubs (Phyllophaga) of economic importance in Central America. Tropical Pest Managernent 30(1): 36-50.

KING, A.B.S. 1985. Factors affecting infestation by larvae of Phyllophaga spp. (Col: Scarabaeidae) in Costa Rica. BulL Ent. Res 75, 417-42.
LOPEZ, R.; SALAZAR, L. 1987. Nematodos asociados al arroz (Oryza sativa L.) en Costa Rica. III. Microscopía electrónica de rastreo de Meloidogyne y Tylenclorhyncus annulatus. Turrialba 37(1):77-84.

MORON, M.A. 1986. El género Phyllophaga en México. Morfología, distribución y sistemática supraespecífica (Insecta: Coleoptera). Instituto de Ecología de México, D.F. $121-/ 45$ p.

MORON, M.A.; TERRON, R.A. 1988. Entomología práctica. Instituto de Ecología, AC. México. 57-86 p.

MORON, M.A. 1988. Las especies de Phyllophaga (Col: Melolonthidae) con mayor importancia agrícola en México. In: III Mesa redonda sobre plagas del suelo. Michoacán, México. 81 -102 p.

SOIL SCIENCE ST AFF. 1994. Keys of taxonomy of soils.

STEHR, F. 1987. Immature Insects. Kendall/Hunt. Iowa, U.S.A. 7-18 p.

TOSI, J.A. 1969. Mapa Ecológico de Costa Rica. Centro Científico Tropical. San José, Costa Rica. 